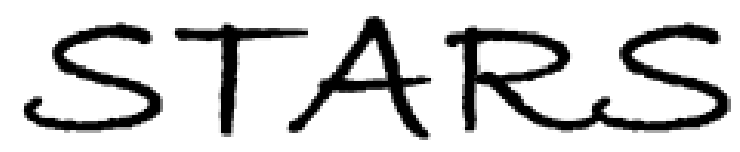

University of Central Florida

STARS

$1-1-2007$

\title{
Low-threshold and high efficiency lasing upon band-edge excitation in a cholesteric liquid crystal
}

\author{
Yuko Matsuhisa \\ University of Central Florida \\ Yuhua Huang \\ University of Central Florida \\ Ying Zhou \\ University of Central Florida \\ Shin-Tson Wu \\ University of Central Florida \\ Ryotaro Ozaki
}

Find similar works at: https://stars.library.ucf.edu/facultybib2000

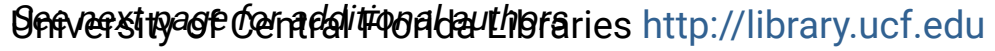

This Article is brought to you for free and open access by the Faculty Bibliography at STARS. It has been accepted for inclusion in Faculty Bibliography 2000s by an authorized administrator of STARS. For more information, please contactSTARS@ucf.edu.

\section{Recommended Citation}

Matsuhisa, Yuko; Huang, Yuhua; Zhou, Ying; Wu, Shin-Tson; Ozaki, Ryotaro; Takao, Yuuki; Fujii, Akihiko; and Ozaki, Masanori, "Low-threshold and high efficiency lasing upon band-edge excitation in a cholesteric liquid crystal" (2007). Faculty Bibliography 2000s. 7412.

https://stars.library.ucf.edu/facultybib2000/7412

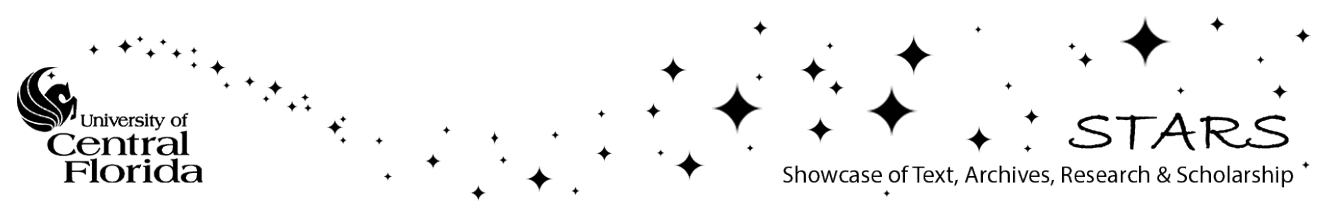




\section{Authors}

Yuko Matsuhisa, Yuhua Huang, Ying Zhou, Shin-Tson Wu, Ryotaro Ozaki, Yuuki Takao, Akihiko Fujii, and Masanori Ozaki 


\section{Low-threshold and high efficiency lasing upon band-edge excitation in a cholesteric liquid crystal}

Cite as: Appl. Phys. Lett. 90, 091114 (2007); https://doi.org/10.1063/1.2710777

Submitted: 09 October 2006 . Accepted: 28 January 2007 . Published Online: 01 March 2007

Yuko Matsuhisa, Yuhua Huang, Ying Zhou, Shin-Tson Wu, Ryotaro Ozaki, Yuuki Takao, Akihiko Fujii, and Masanori Ozaki

\section{ARTICLES YOU MAY BE INTERESTED IN}

The photonic band edge laser: A new approach to gain enhancement

Journal of Applied Physics 75, 1896 (1994); https://doi.org/10.1063/1.356336

How doping a cholesteric liquid crystal with polymeric dye improves an order parameter and makes possible low threshold lasing

Journal of Applied Physics 94, 279 (2003); https://doi.org/10.1063/1.1578534

Cholesteric liquid crystal laser with wide tuning capability

Applied Physics Letters 86, 161120 (2005); https://doi.org/10.1063/1.1897439
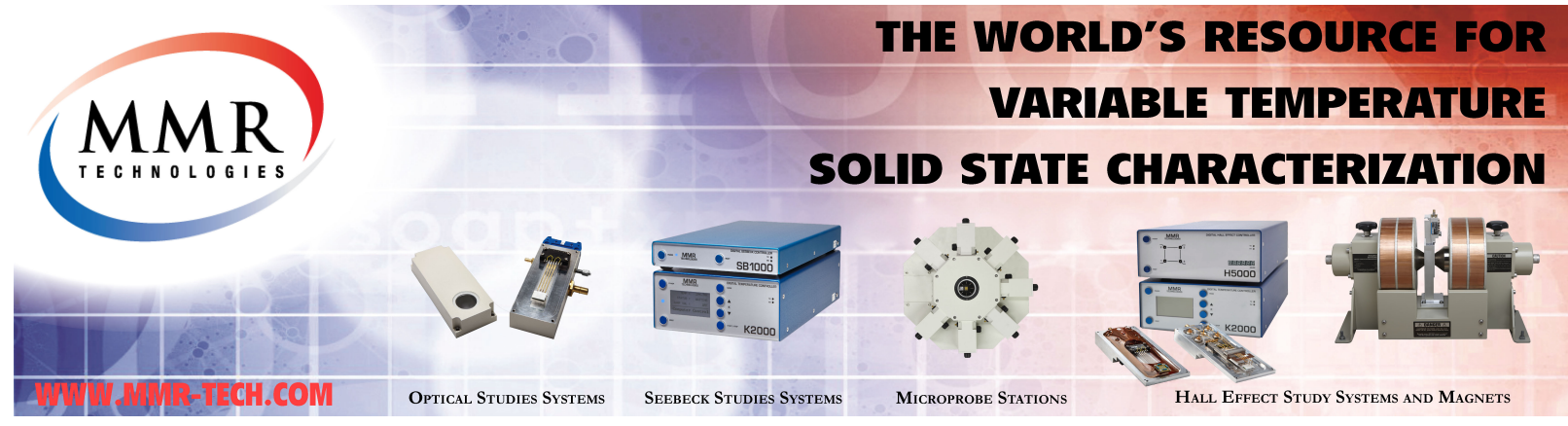


\title{
Low-threshold and high efficiency lasing upon band-edge excitation in a cholesteric liquid crystal
}

\author{
Yuko Matsuhisa, Yuhua Huang, Ying Zhou, and Shin-Tson Wua) \\ College of Optics and Photonics, University of Central Florida, Orlando, Florida 32816 \\ Ryotaro Ozaki \\ Electrical and Electronic Engineering, National Defense Academy, 1-10-20 Hashirimizu, \\ Yokosuka, 239-8686 Japan \\ Yuuki Takao, Akihiko Fujii, and Masanori Ozaki \\ Department of Electrical, Electronic and Information Engineering, Graduate School of Engineering, \\ Osaka University, 2-1 Yamada-Oka, Suita, Osaka 565-0871, Japan
}

(Received 9 October 2006; accepted 28 January 2007; published online 1 March 2007)

\begin{abstract}
A low threshold and high efficiency laser based on dye-doped cholesteric liquid crystals (CLCs) is demonstrated using an input excitation with the same handedness of circular polarization as the helical structure of the sample at the shorter wavelength band edge of the reflection band. The responsible mechanism originates from the dramatic increase of the optical density of state (DOS) at the band edges. The calculated DOS of the CLC system confirms the authors' experimental results. (C) 2007 American Institute of Physics. [DOI: 10.1063/1.2710777]
\end{abstract}

Liquid crystals containing chiral molecules such as cholesteric liquid crystals (CLCs), ferroelectric liquid crystals, cholesteric blue phases, and cholesteric glasses have recently attracted great attention as self-organized photonic crystals. Different from other photonic crystals, the photonic band gap of the CLC is polarization dependent. A circularly polarized light in the opposite handedness to the liquid crystal helix transmits through the CLC, while for the one in the same handedness a photon density of state (DOS) vanishes in the photonic band gap. ${ }^{1,2}$ Since the photon group velocity approaches zero and DOS is enhanced at the edge of the photonic band gap, low-threshold lasers have been theoretically predicted $^{3}$ and experimentally demonstrated. ${ }^{4-16}$ Moreover, an anomalous strong absorption occurs at the band edge of an absorbing CLC. A low-threshold CLC laser upon such phenomena has been theoretically predicted. ${ }^{17}$ So far, however, no experimental demonstration has been carried out.

In this letter, we experimentally demonstrate the lowthreshold laser action upon the band-edge effect due to the anomalous absorption in a dye-doped CLC. To enhance the band-edge effect, we match the pump laser wavelength to the higher energy band edge of the photonic band gap of the dye-doped CLC by controlling the operating temperature. We also perform theoretical calculation about the DOS, which shows good agreement with the experimental result.

The CLC host was prepared by mixing $72.5 \%$ nematic liquid crystal BL006 with $27.5 \%$ of a right-handed chiral dopant MLC6248 (both are from Merck). Afterwards, a $0.1 \mathrm{wt} \%$ laser dye PM597 (exciton) was doped into the mixture. The CLC was filled into a $12-\mu \mathrm{m}$-thick LC cell by capillary action. The inner surfaces of the glass substrates were coated with a thin polyimide alignment layer and rubbed in antiparallel directions. The pretilt angle is $\sim 3^{\circ}$. The helical axis of the CLC was perpendicular to the glass substrates. The temperature of the sample was controlled by a temperature controller.

\footnotetext{
${ }^{a)}$ Electronic mail: swu@mail.ucf.edu
}

To investigate the laser emission properties, a second harmonic laser from a $Q$-switched neodymium-doped yttrium aluminum garnet pulsed laser (MiniliteII, Continuum) with vertical linear polarization was used for the excitation. The wavelength, pulse width, and pulse repetition frequency are $\lambda=532 \mathrm{~nm}, 4 \mathrm{~ns}$, and $1 \mathrm{~Hz}$, respectively. The linear polarized light beam was converted into right-handed circularly polarized (RCP) or left-handed circularly polarized (LCP) light by a quarter-wave plate and was focused by a lens onto the sample. The spot diameter on the sample was measured to be about $160 \mu \mathrm{m}$. The emitted laser light from the sample was collected into a fiber-based spectrometer (Ocean Optics, HR4000).

The dashed lines in Fig. 1(a) show the transmission

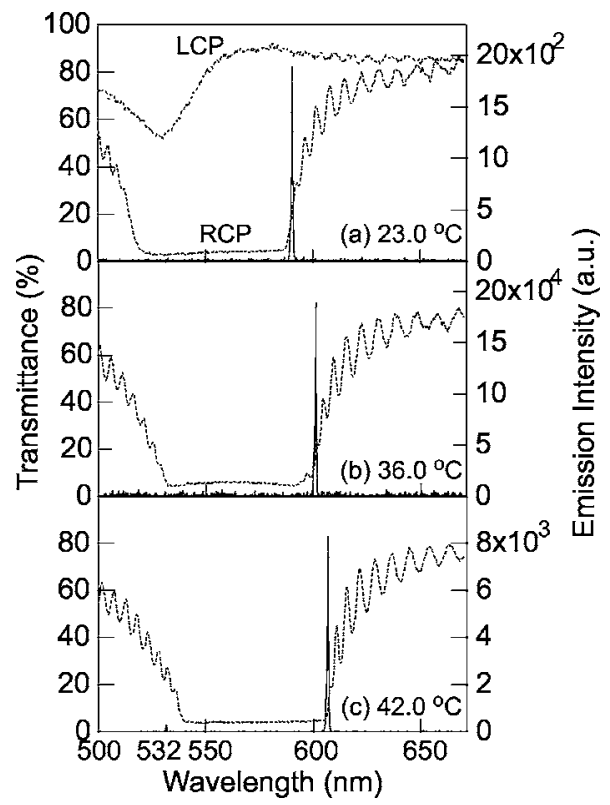

FIG. 1. (a) Transmission spectra of the CLC system for the RCP and LCP incident lights and the emission spectrum at $23.0^{\circ} \mathrm{C}$. [(b) and (c)] Transmission spectra for the RCP incident light and emission spectra at 36.0 and $42.0^{\circ} \mathrm{C}$. 


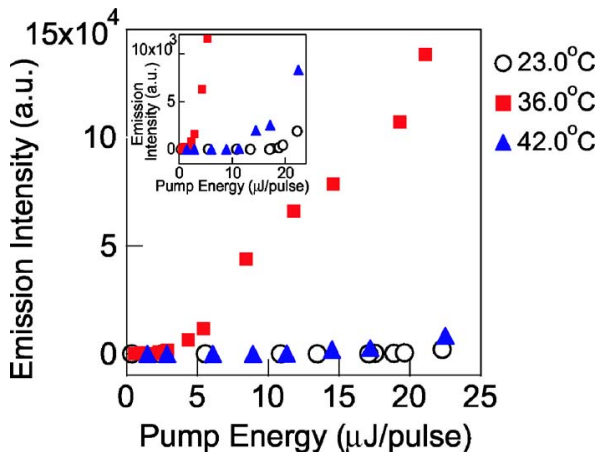

FIG. 2. Emission intensity as a function of pumping energy at 23.0, 36.0, and $42.0^{\circ} \mathrm{C}$.

spectra of the dye-doped CLC for the LCP and RCP incident lasers at $23.0^{\circ} \mathrm{C}$. For the LCP incident light, the absorption of the laser dye was observed at $\lambda \sim 530 \mathrm{~nm}$. On the other hand, for the RCP incident light, the suppressed transmittance in the spectral range from 519 to $590 \mathrm{~nm}$ is due to the stop band of the CLC. The stop band covers the wavelength of the excitation beam $(\lambda=532 \mathrm{~nm})$ at room temperature. The dashed lines in Figs. 1(b) and 1(c) show the transmission spectra for the RCP incident light at 36.0 and $42.0^{\circ} \mathrm{C}$, respectively. The stop band shifts toward the longer wavelength side as the temperature increases, which is attributed to the temperature dependence of the helical pitch length of the CLC. At $36.0^{\circ} \mathrm{C}$, the high-energy band edge is located at the wavelength of the excitation beam, as Fig. 1(b) shows. When the temperature is increased to $42.0^{\circ} \mathrm{C}$, the stop band is shifted to the 540-607 nm spectral range. In this case, the pumping laser wavelength is outside the stop band, as shown in Fig. 1(c).

The solid lines in Fig. 1 show the emission spectra as a function of temperature when the pump energy is $\sim 22 \mu \mathrm{J} /$ pulse. Above a threshold, the laser action was observed at the low-energy band edge. Continue to increasing the temperature, the lasing wavelength shifts to a longer wavelength, which is in accordance with the redshift of the band edge. Figure 2 shows the emission intensities as a function of pumping energy. The lasing efficiency strongly depends on the temperature. As shown in the inset of Fig. 2, the lasing efficiency at $23.0^{\circ} \mathrm{C}$ is approximately 4.4 times less than that at $42.0^{\circ} \mathrm{C}$. From Fig. 1, the stop band overlaps the excitation wavelength and a portion of the pump energy is reflected at $23.0^{\circ} \mathrm{C}$. While at $42.0^{\circ} \mathrm{C}$, since the excitation wavelength is outside the reflection band, all the excitation energy is able to pump the dye molecules. As a result, higher optical gain is achieved in the sample at $42.0^{\circ} \mathrm{C}$ than that at $23.0^{\circ} \mathrm{C}$. Consequently, higher laser efficiency is obtained when the sample temperature is controlled at $42.0^{\circ} \mathrm{C}$.

The most interesting discovery is that the lasing efficiency at $36.0{ }^{\circ} \mathrm{C}$ is further enhanced by $\sim 17$ times as compared to that at $42.0^{\circ} \mathrm{C}$. This is contributed by the band-edge effect. At the edges of the photonic band gap, the DOS and photon dwell time are significantly enhanced because of the multiple internal reflections of the CLC. Therefore, when the band edge is within the absorption spectrum of the laser dye, the excitation of the dye molecules is dramatically enhanced, which means much more dye molecules are elevated to the excited state by anomalous absorption of the pumping photons. As a result, the laser efficiency is dramatically enhanced.

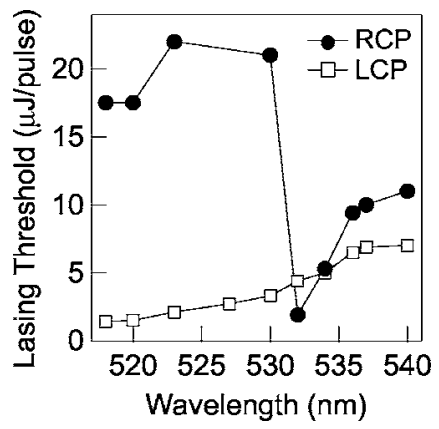

FIG. 3. Lasing threshold as a function of wavelength of high-energy band edge for the LCP and RCP excitation beams.

We also investigated the lasing threshold of the dyedoped CLC sample for the RCP and LCP pumping beams as a function of the wavelength of the high-energy band edge by changing the temperature. The results are shown in Fig. 3. For the LCP excitation beam, the lasing threshold gradually increases with the redshift of the bandedge. This stems from the spontaneous emission spectrum of the doped dye, which determines the optical gain. The dye used in this experiment exhibits its maximum spontaneous emission at $\lambda=580 \mathrm{~nm}$. Therefore, the lasing threshold reaches the lowest when the lasing wavelength occurs at $580 \mathrm{~nm}$ and then gradually increases when the lasing wavelength departs from $\lambda=580 \mathrm{~nm}$.

On the other hand, for the RCP excitation beam the lasing threshold is high when the shorter band edge of the band gap is below the pumping wavelength $\lambda=532 \mathrm{~nm}$. This phenomenon can be explained as follows. At a short wavelength, the stop band covers the wavelength of the excitation beam, as Fig. 1(a) shows. As a result, the incident beam is reflected by the stop band, which causes the lasing threshold to increase. ${ }^{18}$ Note that the lasing threshold is significantly decreased from 21 to $1.9 \mu \mathrm{J} /$ pulse when the shorter band edge is located at the pumping wavelength of $532 \mathrm{~nm}$. Moreover, the lasing threshold for the RCP excitation beam is $\sim 2.3$ times smaller than that for the LCP one. This is contributed by the above-mentioned band-edge effects. When the bandedge wavelength gets longer than $532 \mathrm{~nm}$, the lasing threshold increases again and becomes higher than the threshold for the LCP excitation beam, which is attributed to the reflection induced by interference. From the result, the bandedge excitation is remarkably effective to increase the lasing efficiency and lower the lasing threshold.

To confirm the experimental results, we calculated the DOS of the CLC system ${ }^{19,20}$ using the $4 \times 4$ matrix method. ${ }^{21}$ In our calculations, the thickness and the extraordinary and ordinary refractive indices of the CLC are $12.0 \mu \mathrm{m}$ and 1.700 and 1.506, respectively, according to our experimental conditions. The absorption of the CLC medium is neglected. We investigated the DOS as a function of wavelength of the high-energy band edge for LCP or RCP incident light by changing the helical pitch. As plotted in Fig. 4, the DOS for the LCP incident light is insensitive to the wavelength of the band edge. This is because a circularly polarized light in the opposite handedness to the CLC helix could not interact with the CLC medium. On the other hand, for the RCP incident light, the DOS at a shorter wavelength is smaller than that of the LCP light due to the overlapping with the stop band. The low DOS reduces the optical gain. However, in the vicinities of $\lambda=532 \mathrm{~nm}$ the DOS is drastically 


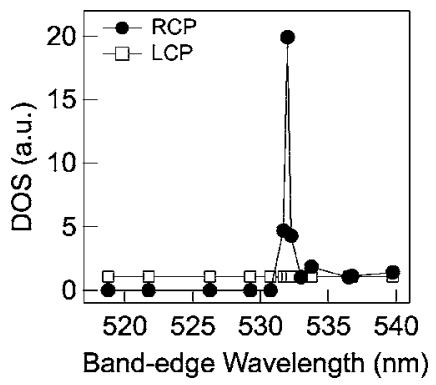

FIG. 4. DOS as a function of wavelength of high-energy band edge for the LCP or RCP incident light.

increased to 20 because the band edge coincides with the pumping wavelength. This high DOS greatly enhances the lasing efficiency and decreases the lasing threshold at $532 \mathrm{~nm}$, as shown in Fig. 3. The calculated results agree well with our experimental observations.

In conclusion, we have demonstrated a low-threshold laser action in dye-doped CLC based on band-edge excitation. The lasing efficiency is dramatically enhanced and the lasing threshold significantly decreased by the band-edge excitation. Our theoretical calculations on the DOS in the CLC confirm our experimental results.

This work is partially supported by Grant-in-Aids for Scientific Research from the Ministry of Education, Culture, Sports, Science and Technology of Japan and JSPS. The UCF part is supported by AFOSR.
${ }^{1}$ E. Yablonovitch, Phys. Rev. Lett. 58, 2059 (1987).

${ }^{2}$ S. John, Phys. Rev. Lett. 58, 2486 (1987).

${ }^{3}$ J. P. Dowling, M. Scalora, M. J. Bloemer, and C. M. Bowden, J. Appl. Phys. 75, 1896 (1994).

${ }^{4}$ V. I. Kopp, Z. Zhang, and A. Z. Genack, Prog. Quantum Electron. 27, 369 (2003).

${ }^{5}$ Y. Huang, Y. Zhou, and S. T. Wu, Appl. Phys. Lett. 88, 011107 (2006).

${ }^{6}$ V. I. Kopp, B. Fan, H. K. M. Vithana, and A. Z. Genack, Opt. Lett. 23, 1707 (1998).

${ }^{7}$ M. Ozaki, M. Kasano, D. Ganzke, W. Haase, and K. Yoshino, Adv. Mater. (Weinheim, Ger.) 14, 306 (2002).

${ }^{8}$ M. Kasano, M. Ozaki, K. Yoshino, D. Ganzke, and W. Haase, Appl. Phys. Lett. 82, 4026 (2003).

${ }^{9}$ M. Ozaki, M. Kasano, T. Kitasho, D. Ganzke, W. Haase, and K. Yoshino, Adv. Mater. (Weinheim, Ger.) 15, 974 (2003).

${ }^{10}$ W. Cao, A. Munoz, P. Palffy-Muhoray, and B. Taheri, Nat. Mater. 1, 111 (2002).

${ }^{11}$ P. V. Shibaev, V. I. Kopp, A. Genack, and E. Hanelt, Liq. Cryst. 30, 1391 (2003).

${ }^{12}$ A. F. Munoz, P. Palffy-Muhoray, and B. Taheri, Opt. Lett. 26, 804 (2001).

${ }^{13}$ Y. Huang, Y. Zhou, and S. T. Wu, Opt. Express 14, 1236 (2006).

${ }^{14}$ S. Furumi, S. Yokoyama, A. Otomo, and S. Mashiko, Appl. Phys. Lett. 82, 16 (2003).

${ }^{15}$ K. Funamoto, M. Ozaki, and K. Yoshino, Jpn. J. Appl. Phys., Part 142 , 1523 (2003).

${ }^{16}$ J. Schmidtke, S. Kniesel, and H. Finkelmann, Macromolecules 38, 1357 (2005).

${ }^{17}$ V. A. Belyakov, Mol. Cryst. Liq. Cryst. 453, 43 (2006).

${ }^{18}$ S. Furumi and Y. Sakka, Adv. Mater. (Weinheim, Ger.) 18, 775 (2006).

${ }^{19}$ J. Schmidtke and W. Still, Eur. Phys. J. B 31, 179 (2003).

${ }^{20}$ M. D. Tocci, M. Scalora, M. J. Bloemer, J. P. Dowling, and C. M. Bowden, Phys. Rev. A 53, 2799 (1996).

${ }^{21}$ D. W. Berreman, J. Opt. Soc. Am. 63, 1374 (1973). 